\title{
Extinction behavior following differential partial reward sequences
}

\section{J. M. BLOOM, Texas Research Institute}

In three experiments, rats were trained in the straight runway on NRR or RNR partial reward sequences and then extingtuished. Contrary to prediction from the stimulus aftereffects model, RNR produced greater resistance to extinction.

According to the stimulus aftereffects conditioning model (Capaldi, 1957). Ss trained on different sequences of rewarded (R) and nonrewarded trials $(\mathrm{N})$ should be differentially resistant to extinction. All other things equal, NR sequences should produce the greatest resistance, $R R$ sequences less, and $R N$ sequences least resistance.

However, Spivey and his associates (Spivey \& Hess, 1968: Spivey, Hess, \& Black, 1968) twice trained rat Ss on RRNN and RRRR sequences and found no differences in extinction. The present experiments compared the effects of $R N$ vs $R R$ sequences in rat $S s$ trained on NRR and RNR patterns. The aftereffects model predicts greater resistance to extinction for the NRR than the RNR condition.

\section{EXPERIMENT 1}

Subjects

The Ss were 24 250-300 g naive male albino rats purchased from the Holtzman Co.. Madison, Wisconsin.

Apparatus

The apparatus was a gray wooden straight runway, $60 \mathrm{in.} \mathrm{long.}$ $4 \mathrm{in}$. wide, and $6 \mathrm{in}$. high with mesh bottom and Plexiglas top. At the goal end was a sliding food tray mounted behind a gray 2 -in. high wooden barrier. A partition divided the tray into two compartments, and it could be aligned so that only one compartment was accessible to $\mathrm{S}$.

Infrared photocell units were mounted 6 in. and 54 in. from the start end of the runway. When $S$ interrupted the final photobeam an electric brake was released, which allowed a counter-weighted 5-1/2 in. section of the Plexiglas roof to swing down, forming a 14 in. goal box. The brake was then automatically reactivated, locking the door in place and confining $S$ to the goal area. A Standard Electric 1/100 sec clock measured time between placing
$S$ in the runway through the first photobeam and S's interruption of the final photobeam. The runway was also equipped to measure start, run, and goal times, but these data were not included in the analysis.

Procedure

On the first 14 days the Ss were fed Rockland Breeder diet pellets for $1 \mathrm{~h}$ at the approximate time that they were to receive the daily training trials. On Days 13 and 14 , the Ss were placed in the goal box for the first $5 \mathrm{~min}$ of the feeding period with the food tray containing $.045 \mathrm{~g}$ Noyes pellets.

Following pretraining. Group NRR (12 Ss) received a nonrewarded, rewarded, and rewarded trial each day, in that order, for 14 days. Group RNR (12 Ss) received similar training except that the sequence was reward, nonreward. reward. The Ss were run in alternating order between groups. Following the last acquisitjon day, each $\mathrm{S}$ was given two days of extinction triils, 15 on the first and 12 on the second day.

Each acquisition trial ended with $40 \mathrm{sec}$ goal confinement and each extinction trial $10 \mathrm{sec}$ confinement. Between trials. $S$ was placed in a neutral box for $30 \mathrm{sec}$. The regular 1-h feeding was given about $15 \mathrm{~min}$ after the last daily trial.

\section{RESULTS AND DISCUSSION}

The results are shown in Fig. 1. On the last day of acquisition, both groups responded appropriately to the amount of reward on a particular trial, Group NRR running slow-fast-fast and Group RNR running fast-slow-fast. Patterning was more pronounced in NRR, but responding on trials following nonreward was the same in both groups.

These tendencies were also evident in the initial extinction phase, Group NRR responding slower on Trial 1, but both groups responding at the same level on the first and second trials following nonreward. Thereafter, Group NRR ran slower than RNR. Day 2 of extinction produced essentially the same results. It is of interest that 15 extinction trials did not alter the initial phase response tendencies. Group NRR responded slower on the first trial than RNR, and both groups responded at the same level on Trials 2 and 3.

With respect to the aftereffects model, the extinction results

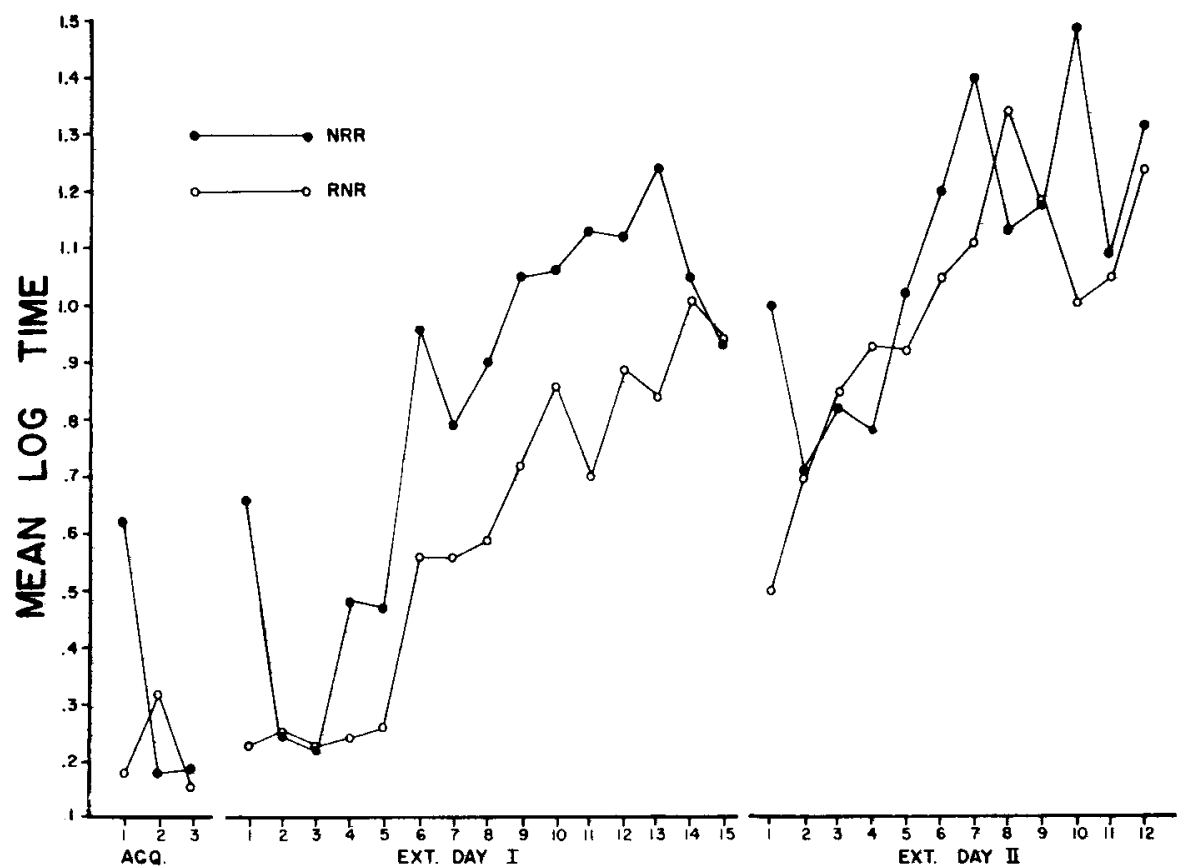

TRIALS
Fig. 1. Mean log running time on each trial of the last acquisition day and the extinction days of Experiment 1. 


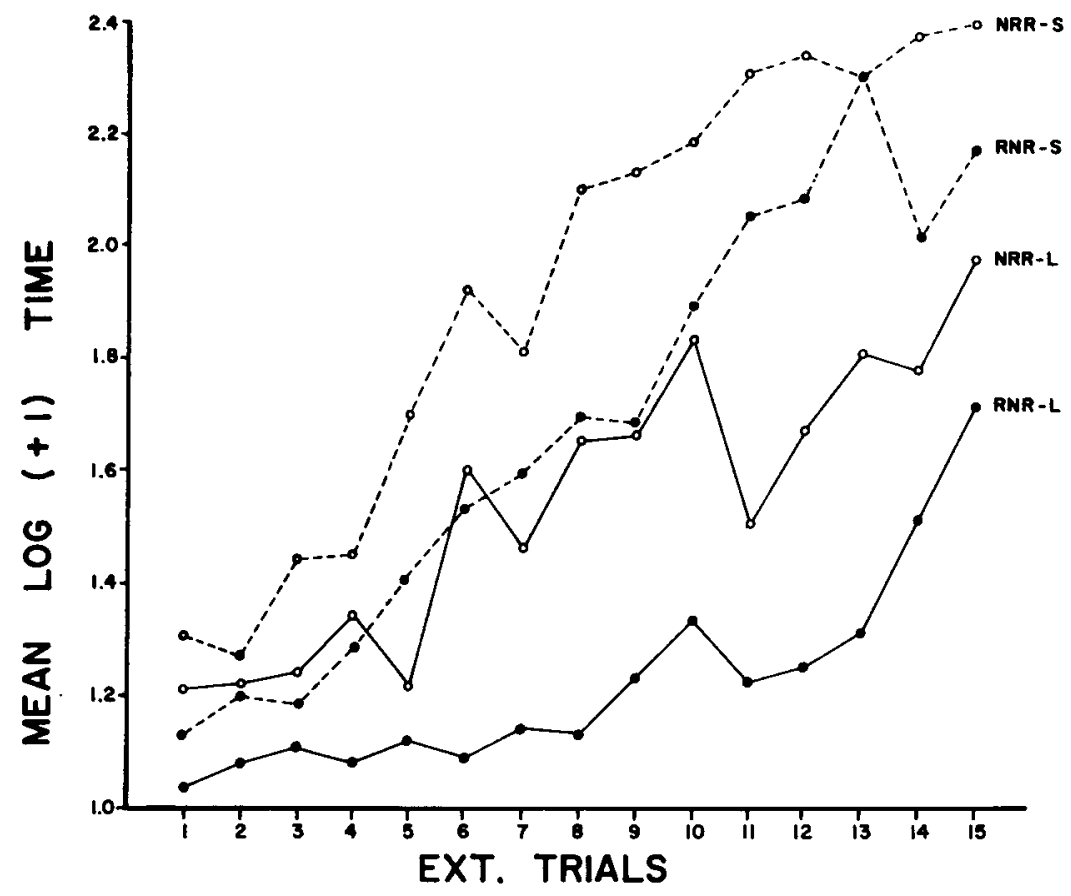

Fig. 2. Mean log running time in Experiments 2 and 3 for long and short intertrial interval groups.

were contrary to prediction. Since the prediction was directional, it was felt that statistical analysis would be inappropriate.

However, the data were carefully inspected for differences during acquisition which might have produced the observed relationship during extinction. The most obvious difference was the fact that NRR Ss ran slower on the first trial of the day than RNR Ss. Capaldi (personal communication) has pointed out that the NRR Ss, anticipating nonreward, may not have registered the first trial as a nonreward. And while the RNR Ss also anticipated nonreward, patterning was more pronounced in NRR.

Accordingly, Experiments 2 and 3 were designed to prevent patterning during acquisition. Secondary purposes were to control for possible between-trial differential odor traces (cf., McHose \& Ludvigson, 1967), and to determine the effects of lengthening the intertrial interval.

Subjects

\section{EXPERIMENTS 2 and 3}

The Ss were 32 250-300 g naive male albino rats purchased from the Holtzman Co., Madison, Wisconsin. One S from Group NRR-L (Experiment 3) died during the experiment.

Apparatus

The apparatus used in Experiment 1 was modified by the addition of an exhaust blower installed underneath the goal area with intake in the start area. It was estimated that the air in the goal area was completely exchanged about every $20 \mathrm{sec}$.

Procedure

Pretraining and training were exactly the same as in Experiment 1 with the following exceptions. In Experiment 2, there were 18 days of acquisition training. Groups NRR-S (five Ss) and RNR-S (five Ss) received the sequences as indicated except that on Days $3,7,10$, and 14 each $S$ received a single rewarded trial and on Days 4, 8, 13, and 17 a single nonrewarded trial. On Day 19 of training, each $S$ received 15 extinction trials. The intertrial interval was $30 \mathrm{sec}$ and goal box confinement was $30 \mathrm{sec}$ throughout training.
Part of Experiment 3 was a replication of Experiment 2 with three Ss per group. In addition, Groups NRR-L (seven Ss) and RNR-L (eight Ss) were trained exactly as in the $S$ conditions except that the Ss were run in rotating order. All Ss received the first trial of the day before any $S$ received Trial 2 , and so on, creating an intertrial interval of at least $20 \mathrm{~min}$. Again, the Ss were run in alternating order between groups, the effect being to produce exactly the same between-S trial sequences in each $L$ group-one NR, one RN, and one RR on three-trial days and either NN or RR on one-trial days.

\section{RESULTS AND DISCUSSION}

There was no evidence of patterning in either experiment. The extinction results are shown in Fig. 2 . In both the $L$ and $S$ conditions Group NRR ran slower than Group RNR on the first trial, but the differences were not significant (for $L, F=2.70$, $\mathrm{df}=1 / 13$; for $\mathrm{S}, \mathrm{F}=2.86, \mathrm{df}=1 / 14)$. In both intertrial interval conditions, the NRR pattern produced slower responding during extinction than the RNR pattern.

It is evident that either RN sequences increase resistance to extinction in the RNR condition relative to NRR, or RR sequences decrease resistance in NRR relative to RNR. Neither effect is predicted by the aftereffects model.

\section{REFERENCES}

CAPALDI, E. J. A sequential hypothesis of instrumental learning. In K. W. Spence and J. T. Spence (Eds.), The psychology of learning and motivation. Vol. 1. New York: Academic Press, 1967.

McHOSE, J. H., \& LUDVIGSON, H. W. Differential conditioning with nondifferential reinforcement. Psychonomic Science, 1966, 6, 485-486.

SPIVEY, J. E., \& HESS, D. T. Effect of partial reinforcement trial sequences on extinction performance. Psychonomic Science, 1968, 10, 375-376.

SPIVEY, J. E., HESS, D. T., \& BLACK, D. Influence of partial reinforcement pattern and intertrial reinforcement on extinction performance following abbreviated training. Psychonomic Science, 1968, 10, 377-378.

$$
\text { NOTE }
$$

1. Address: 1300 Moursund, Houston, Texas 77025. 